\title{
Unlocking the secrets of the midplane gas and dust distribution in the young hybrid disc HD 141569
}

\author{
J. M. Miley ${ }^{1}$, O. Panić ${ }^{1}$, M. Wyatt ${ }^{2}$, and G. M. Kennedy ${ }^{3,4}$ \\ 1 School of Physics and Astronomy, University of Leeds, Woodhouse Lane, Leeds LS2 9JT, UK \\ e-mail: py12 jm@leeds.ac.uk \\ 2 Institute of Astronomy, University of Cambridge, Madingley Road, Cambridge CB3 OHA, UK \\ 3 Department of Physics, University of Warwick, Gibbet Hill Road, Coventry CV4 7AL, UK \\ ${ }^{4}$ Centre for Exoplanets and Habitability, University of Warwick, Gibbet Hill Road, Coventry CV4 7AL, UK
}

Received 6 May 2018 / Accepted 20 June 2018

\begin{abstract}
Context. HD 141569 is a pre-main sequence star with a disc uniquely placed between protoplanetary and debris discs, similar to the older "hybrid" type discs.

Aims. This work aims to place the mass and spatial structure of the disc midplane in the context of the debris, hybrid and protoplanetary discs.

Methods. We observed HD 141569 with ALMA in $1.3 \mathrm{~mm}$ continuum and ${ }^{13} \mathrm{CO}(2-1)$. This is the first detection and image of the optically thin gas emission from the midplane of this disc.

Results. In continuum emission, we detect a combination of an unresolved central peak and a ring of millimetre emission at $220 \pm 10 \mathrm{au}$, slightly interior to one of the rings discovered in scattered light. The minimum dust mass of the ring is $0.13 \pm 0.02 M_{\oplus}$ while the unresolved millimetre peak at the stellar location is predominantly thermal emission due to a minimum of $1.2 \pm 0.2 M_{\oplus}$ of dust. ${ }^{13} \mathrm{CO}$ is distributed asymmetrically around the stellar position with a peak at $1{ }^{\prime \prime} 1$ distance and a PA of $-33^{\circ}$. The gas is detected as far as $220 \pm 10 \mathrm{au}$, a radial separation the same as that of the mm ring. Assuming optically thin emission and standard ISM abundances, we used our ${ }^{13} \mathrm{CO}$ data to derive the gas mass in the disc of $(6.0 \pm 0.9) \times 10^{-4} M_{\odot}$. Comparison to published ${ }^{12} \mathrm{CO}$ data shows that ${ }^{12} \mathrm{CO}$ is optically thick, explaining why estimates based on ${ }^{12} \mathrm{CO}$ underestimated the gas mass.
\end{abstract}

Key words. protoplanetary disks - planets and satellites: rings - stars: variables: T Tauri, Herbig Ae/Be

\section{Introduction}

Understanding the evolution from protoplanetary to debris discs (Dominik \& Dullemond 2002; Wyatt et al. 2015) requires an understanding of their structures. How do the many gaps, dust traps and asymmetries seen in protoplanetary discs evolve into the one or two dusty debris rings, and what is the role of gas in this process? The "hybrid" discs (Péricaud et al. 2017), are believed to probe this step in evolution because of detectable amounts of gas in the disc despite otherwise appearing to have evolved beyond the protoplanetary disc limit in dust mass.

Herbig star HD 141569 hosts the only known hybrid disc amongst pre-main sequence stars to date, a B9-A0 type, $5 \mathrm{Myr}$ old star at a Gaia distance of $111 \pm 1$ pc (Weinberger et al. 2000; Lindegren et al. 2016) ${ }^{1}$. The spectral energy distribution (SED) and IR excess luminosity of 0.0084 (Sylvester et al. 1996) were the first indicators of this unique status. HD 141569 has an SED which appears as a scaled-down version of a protoplanetary disc SED, clearly dominated by multiple radial (temperature) components, discerning it from the known debris and hybrid discs, as both these types show, at most, two radial (temperature) components (e.g., 49 Ceti, HD 131835, Roberge et al. 2013; Moór et al. 2015; Kennedy \& Wyatt 2014). The dust mass of

\footnotetext{
1 We have scaled previously reported length scales to the new Gaia distance for the source.
}

$0.7 M_{\oplus}$ (Sandell et al. 2011; Panic et al. 2013) places HD 141569 between the protoplanetary and debris-disc regimes (Wyatt et al. 2015). First detected by Dent et al. (2005), the CO gas extends up to $200 \mathrm{au}$ (Flaherty et al. 2016; White et al. 2016) and is nested inside the two outermost rings detected by Hubble Space Telescope NICMOS at 234 and 388 au (Augereau et al. 1999; Weinberger et al. 1999; Biller et al. 2015). Reported gas masses range between $4.5 \times 10^{-6} M_{\odot}$ derived from ${ }^{12} \mathrm{CO}$ (White et al. 2016) and $5 \times 10^{-4} M_{\odot}$ derived from SED model fits to Herschel line observations (Thi et al. 2014). None of these estimates employed optically thin $\mathrm{CO}$ isotopologue emission, which until now remained undetected in this disc. The estimated gas mass range for HD 141569 is below the typical protoplanetary disc gas masses of $10^{-3} M_{\odot}$ to $10^{-1} M_{\odot}$ but above the hybrid discs gas masses which are mainly $<10^{-6} M_{\odot}$ (Péricaud et al. 2017).

Insight into the innermost regions comes from SED fitting, with an inner radius set at $30 \mathrm{au}$ (Marsh et al. 2002), and CO ro-vibrational emission placing gas as close as 11 au from the star (Goto et al. 2006). These regions have recently been imaged with SPHERE on the Very Large Telescope (VLT), detecting a series of concentric, but discontinuous, scattered light "ringlets" at separations of 45, 61 and 89 au (Perrot et al. 2016). Inside these ringlets is a dust disc component within 0 '.2, detected in $8.2 \mu \mathrm{m}$ imaging with VLT VISIR instrument (Perrot et al. 2016). A central, unresolved component of millimetre 
continuum emission peaks at the stellar location (Marsh et al. 2002; White et al. 2018). Beyond the gas-rich region are the two scattered light rings at 234 and $388 \mathrm{au}$, with inclination and PA similar to that of the inner rings seen with SPHERE (Perrot et al. 2016), but showing more complex structures and spirals, possibly due to planets within the disc (Wyatt 2005) or a fly-by (Reche et al. 2009).

In this letter we show to what extent the midplane gas and dust of HD 141569 exhibit protoplanetary and debris disc characteristics, in the context of increasing evidence for a hybrid class of discs.

\section{Observations and results}

Our ALMA observations in Band 6 were taken on 2016, May 16 (PI Panić, ID 2015.1.01600.S). 39 antennas of $12 \mathrm{~m} \mathrm{di-}$ ameter were employed in a configuration providing baseline lengths ranging from $16 \mathrm{~m}$ to $640 \mathrm{~m}$. The velocity resolution is $0.166 \mathrm{~km} \mathrm{~s}^{-1}$ for ${ }^{13} \mathrm{CO}$ and $0.333 \mathrm{~km} \mathrm{~s}^{-1}$ for $\mathrm{C}^{18} \mathrm{O}$, providing optimal sensitivity to the tenuous and previously undetected midplane gas emission. The total effective continuum bandwidth was $4 \mathrm{GHz}$. The data were flagged and calibrated following the ALMA-pipeline data reduction. Titan was used as the flux calibrator, J1550+0527 and J1733-1304 were used as the bandpass and phase calibrators respectively ${ }^{2}$. Using CASA 4.7.1, we subtracted the continuum emission in the uv-space. Images were created using the clean algorithm (Rau \& Cornwell 2011); as we have no a priori knowledge of the distribution of ${ }^{13} \mathrm{CO}$ or indeed of the dust emission, we adopted natural weighting to prioritise the collection of signal, rather than the increased resolution that could be achieved with other baseline weightings. This produced a synthesized beam size of 0 ".65 (72 au) for the continuum and 0 '.71 (79 au) for ${ }^{13} \mathrm{CO}$ and $\mathrm{C}^{18} \mathrm{O}$.

$\mathrm{Rms}$ noise level in the continuum image is $0.11 \mathrm{mJy}^{\text {beam }}{ }^{-1}$, and in the ${ }^{13} \mathrm{CO}$ and $\mathrm{C}^{18} \mathrm{O}$ channel maps rms was 0.04 and $0.03 \mathrm{Jy} \mathrm{km} \mathrm{s}^{-1}$ beam $^{-1}$ respectively. In the continuum imaging an unresolved point source of $1.7 \mathrm{mJy}$ is detected. ${ }^{13} \mathrm{CO}$ does not show a firm detection in the individual channel maps, but the integrated intensity map over the known range of velocities in which ${ }^{12} \mathrm{CO}$ is seen (Flaherty et al. 2016; White et al. 2016) shows a $6 \sigma$ detection in ${ }^{13} \mathrm{CO} . \mathrm{C}^{18} \mathrm{O}$ is not detected $>3 \sigma$ in either the channels or the integrated intensity map.

We perform azimuthal averaging in radial bins of 0 '.12 for the ${ }^{13} \mathrm{CO}$ and $1.3 \mathrm{~mm}$ maps, and $0^{\prime \prime} .15$ for ${ }^{12} \mathrm{CO}$. Neither the strongly asymmetric ${ }^{13} \mathrm{CO}$ nor the unresolved central $1.3 \mathrm{~mm}$ emission can provide reliable constraints on the position angle and inclination of the disc, so for deprojection of coordinates we adopt the values of the ${ }^{12} \mathrm{CO}$ disc, $\mathrm{PA}=-9^{\circ}$ and inclination $=55^{\circ}$ (White et al. 2016). Figure 1 shows the averaged profiles. At 220 au we detect a thin concentration of millimetre grains concentrated into a ring and detect ${ }^{13} \mathrm{CO}(2-1)$ emission as far as $220 \pm 10$ au.

To derive uncertainties for the radial flux profiles we azimuthally average emission free-regions of the image that are sufficiently far from the central emission but close enough to have high beam efficiency. The mean value across emission-free regions from different parts of the image gives a flux profile that describes the background noise in the image as a result of the averaging process. This method is more robust than simply taking the variation in an elliptical bin and does not rely on any predefined disc models. The resultant noise curves are shown in Fig. 1. for each tracer, where the outer belt is detected at $\sim 6 \sigma_{\mathrm{mm}}$

2 To analyse our data we used the available ${ }^{12} \mathrm{CO}(3-2)$ data published in White et al. (2016).

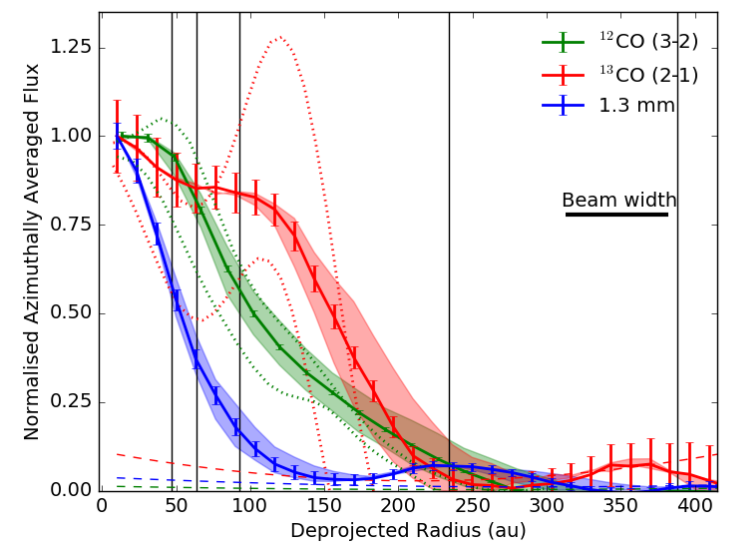

Fig. 1. Azimuthally averaged flux profile following the deprojection of coordinates from the $1.3 \mathrm{~mm}$ image of HD 141569 (blue), ${ }^{12} \mathrm{CO}$ (green) and ${ }^{13} \mathrm{CO}$ (red). Vertical black lines show the radial locations of rings identified in scattered light. Peak intensities for $1.3 \mathrm{~mm}$ continuum, ${ }^{12} \mathrm{CO}$ and ${ }^{13} \mathrm{CO}$ are $1.74 \mathrm{mJy}_{\text {beam }}{ }^{-1}, 1.06$ and $0.25 \mathrm{Jy} \mathrm{km} \mathrm{s}^{-1}$ beam ${ }^{-1}$ respectively. Coloured dotted lines show NW (upper) and SE (lower) cuts of the ${ }^{12} \mathrm{CO}$ and ${ }^{13} \mathrm{CO}$ maps along the position angle of the ${ }^{13} \mathrm{CO}$ peak. The shaded regions show the variation of the profile when changing inclination of the source by $\pm 10^{\circ}$. Error bars are calculated as described in the text, the coloured dashed lines represent the noise level after averaging for each curve.

in contiguous radial bins (comparing the $1.3 \mathrm{~mm}$ curve to the blue dashed noise line), with a peak at $220 \pm 10$ au.

\section{Discussion}

\subsection{Dust emission}

The comparison of the radial profile of $1.3 \mathrm{~mm}$ flux with the known disc structures shows that some of the flux in the central unresolved component may be arising from the rings detected by SPHERE (Perrot et al. 2016). We calculated a minimum dust mass for the inner disc by assuming optically thin emission, using $M_{\text {dust }}=\frac{F_{v} \mathrm{~d}^{2}}{\kappa_{v} B_{v}(T)}$, taking a midplane disc temperature of $20 \mathrm{~K}$, an opacity, $\kappa_{v}$, corresponding to $\mathrm{mm}$ size grains of $1.15 \mathrm{~cm}^{2} \mathrm{~g}^{-1}$ (Draine 2006) and a distance to the star, $\mathrm{d}$, of $111 \mathrm{pc}$. The minimum dust mass for the inner disc is calculated to be $(3.6 \pm 0.5) \times 10^{-6} M_{\odot}=1.2 \pm 0.2 M_{\oplus}$. We have assumed in this calculation that all emission is thermal. A maximum opacity has been chosen to derive a minimum dust mass, the uncertainties correspond to a $\pm 15 \%$ flux calibration uncertainty applied to all ALMA observations. This mass depends on disc temperature, $20 \mathrm{~K}$ is a good assumption for cool material in the midplane and outer disc, increasing the assumed midplane temperature to $50 \mathrm{~K}$ would give a mass of $0.04 M_{\oplus}$

An alternative explanation for the origin of this flux may be free-free emission, rather than thermal emission originating from rings of dust within the inner disc. In fact, a typical mm contribution from free-free emission in Herbig discs at a similar distance to HD 141569, is a few mJy (e.g., Wright et al. 2015), similar to the $1.7 \mathrm{mJy}$ integrated flux we measure for the unresolved peak. Free-free emission has a characteristic spectral slope of -0.1 for optically thin emission, considerably lower than that of thermal continuum emission in this regime and so its effect may be seen in the spectral index. Protoplanetary discs typically show $\alpha_{\mathrm{mm}} \approx 2-3$, transition discs tend to show slightly higher values than this (Pinilla et al. 2014), whilst debris discs have $\alpha_{\mathrm{mm}} \approx 2.5-3$ (MacGregor et al. 2016). ALMA and VLA observations derive a spectral index for the disc of $\alpha_{\mathrm{mm}}=1.63$ 
(MacGregor et al. 2016; White et al. 2018), with which our $1.3 \mathrm{~mm}$ integrated flux of $1.8 \mathrm{mJy}$ is consistent. $\alpha_{\mathrm{mm}}$ calculated between ALMA observations at $870 \mu \mathrm{m}$ and $2.9 \mathrm{~mm}$ also give a steeper, but still relatively low value of 1.81 (White et al. 2018). VLA observations show a significant variability in the continuum flux of the disc, varying by a factor of two to three on timescales of tens of minutes (White et al. 2018). Fluxes measured on integration times shorter than the variability timescale may therefore only offer a snapshot of flux emission. To make a conservative estimate of possible free-free contribution we extrapolated from VLA flux of $54 \mu \mathrm{Jy}$ (MacGregor et al. 2016) using a characteristic spectral slope for optically thin free-free emission; from this estimate the free-free flux at $1.3 \mathrm{~mm}$ would be just $3 \%$ of the total integrated flux we measure, meaning the flux we detect is predominantly thermal, potentially with small free-free contribution of order $10 \mathrm{~s}$ of $\mu \mathrm{Jy}$ at most.

In Fig. 1 we see an increase in measured flux which peaks at a deprojected radial separation of $220 \pm 10 \mathrm{au}$. The ring is detected interior to scattered light ring at 234 au (Biller et al. 2015) as is demonstrated by the ellipses in Fig. 2. Taking the flux from the peak of the ring in Fig. 1 and assuming the same temperature and opacity as before, we are able to calculate a minimum dust mass of $0.13 \pm 0.02 M_{\oplus}$, firmly in the debris disc regime $<1 M_{\oplus}$. We can place an upper limit on the outermost scattered light ring at 388 au from our non-detection in the continuum. Making the same assumptions as before, the maximum mass of the outer ring at which it could still avoid detection in azimuthal averaging is $\sim 0.11 \pm 0.02 M_{\oplus}$.

The position of the millimetre ring relative to the gas disc hints at potential formation mechanisms. A continuum ring at the edge of the gas disc is a morphology that can be compared with models of a primordial, but depleted gas disc. In this case radiation pressure overcomes radial drift to push dust particles of $<100 \mu \mathrm{m}$ outwards to the edge of the gas disc (Takeuchi \& Artymowicz 2001). The ring emission we detect is likely to come from larger grain sizes; millimetre continuum is emitted most efficiently by a narrow range between $1 \mathrm{~mm}$ and $1 \mathrm{~cm}$ (Takeuchi \& Lin 2005). If blow-out from radiation pressure formed the ring, the small grains collecting up at the edge of the gas disc would have to collide and grow into mm-sized grains. This seems unlikely; some small grains will acquire an eccentricity from this process, resulting in collisions that would occur at higher velocity leading to fragmentation rather than grain growth (Takeuchi \& Artymowicz 2001, see Fig. 7). Given our results, a more detailed application of this model to HD 141569 is required, but the level of necessary grain growth seems extremely difficult to achieve. An alternative scenario might be the sculpting of grains into a ring as a result of an unseen planet or perhaps a flyby. Two M dwarfs in a binary system are located less than 9'.0 away from HD 141569 and previous studies of the disc considering a flyby have been able to reproduce global disc features such as the wide gap in the dust and spiral structures (Augereau \& Papaloizou 2004; Ardila et al. 2005; Reche et al. 2009). The caveat on this explanation is that the statistical likelihood of such a flyby is still low and the relationship, both present and past, between HD 141569 and the binary is not completely understood (Reche et al. 2009).

\subsection{Gas emission}

The ${ }^{13} \mathrm{CO}$ emission is strongly asymmetrical (Fig. 2). The emission peak is measured at $6 \sigma_{13}$ off centre from the stellar position, located at a vdeprojected separation of $11^{\prime \prime} 1(120 \pm 10 \mathrm{au})$ at a position angle of $-33^{\circ}$. Whilst at the same separation towards the south-east direction, emission is measured $<2 \sigma_{13}$. The emission we detect from ${ }^{13} \mathrm{CO}$ is roughly co-spatial with the scattered

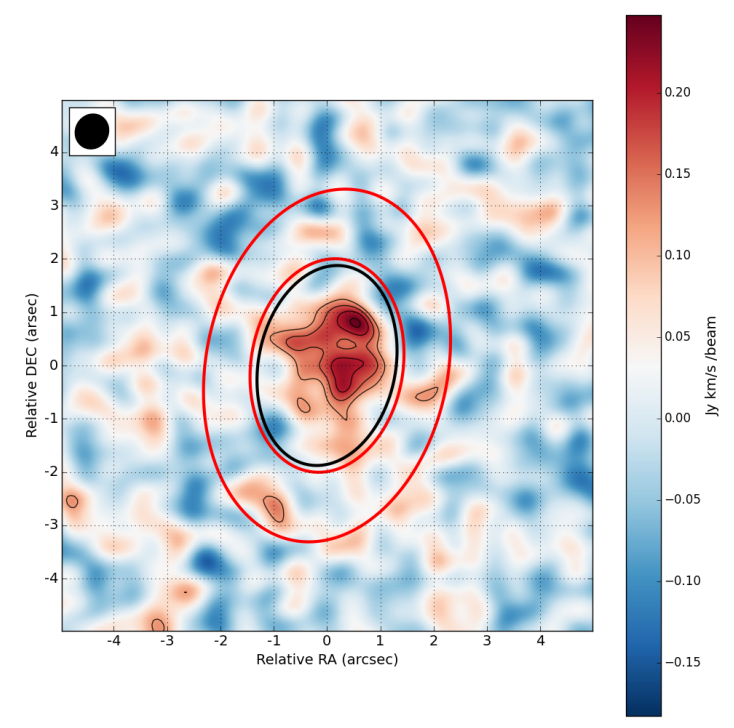

Fig. 2. ${ }^{13} \mathrm{CO}$ emission is shown in the colour map and contoured at levels of $(3,4,5,6) \times \sigma_{13}$, with $\sigma_{13}=0.040 \mathrm{Jy} \mathrm{km} \mathrm{s}^{-1}$ beam $^{-1}$. The red ellipses show the position of the HST rings with parameters from Biller et al. (2015). The black ellipse shows the location of the $\mathrm{mm}$ ring detected in the $1.3 \mathrm{~mm}$ continuum image.

light emission of the eccentric dust ring found at a separation of 89 au (Perrot et al. 2016, their Fig. B.3). Intriguingly, the ring is brighter in scattered light at the opposite side of the disc. ${ }^{13} \mathrm{CO}$ is detected over a considerably larger radial range than the inner edge of the gas disc at 11 au seen in CO rovibrational emission (Goto et al. 2006), than the inner edge of the dust disc of $30 \mathrm{au}$ derived from the SED (Marsh et al. 2002), and than the asymmetry seen in ${ }^{12} \mathrm{CO}$ channel maps (White et al. 2016).

The radially brightest ${ }^{13} \mathrm{CO}$ emission is within the same range of stellocentric distances in which the scattered light rings were found, at 45, 61 and 89 au (Perrot et al. 2016), and cospatial with the HST detection of scattered light between 0 '.4 and 1'.0 (Konishi et al. 2016). Through azimuthal averaging (Fig. 1) we find that the largest radial distance ${ }^{13} \mathrm{CO}$ is detected to is $220 \pm 10 \mathrm{au}$. This separation is coincident with the location of the ring we detect in millimetre emission, with both interior to the 234 au scattered light ring.

Such an asymmetry in molecular gas has been seen in the debris disc Beta Pic (Dent et al. 2014), which may have resulted from giant collisions (for example Jackson et al. 2014), but which is more likely to be due to the collisional destruction of planetesimals trapped in resonance with a planet (Wyatt 2003; Matrà et al. 2017). This scenario would make the gas secondary and so may be different to HD 141569 if the gas in the latter is primordial; the large radial extent of the gas is more typical of a primordial origin, but the nature of the gas in the disc is yet to be confirmed. Younger, protoplanetary, discs can also exhibit strong azimuthal asymmetry that is typically strongest in the dust emission, for example, Oph IRS 48, MWC 758 (van der Marel et al. 2013; Boehler et al. 2017). "Lop-sided" discs form as a result of dust traps that depend upon the surface density and turbulence of the disc (Pinilla et al. 2012; Birnstiel et al. 2013), making it an unlikely mechanism in the less massive hybrid and debris discs.

To determine the optical depth of ${ }^{12} \mathrm{CO}$, we calculated the spatial distribution of $\tau_{12} \mathrm{CO}$ by using the ${ }^{12} \mathrm{CO}$ and ${ }^{13} \mathrm{CO}$ images. We converted the higher transition ${ }^{12} \mathrm{CO}(3-2)$ emission into the minimum expected ${ }^{12} \mathrm{CO}(2-1)$ emission using a ${ }^{12} \mathrm{CO}(2-1) /$ ${ }^{12} \mathrm{CO}(3-2)$ ratio (1.08) obtained from the RADEX online tool 


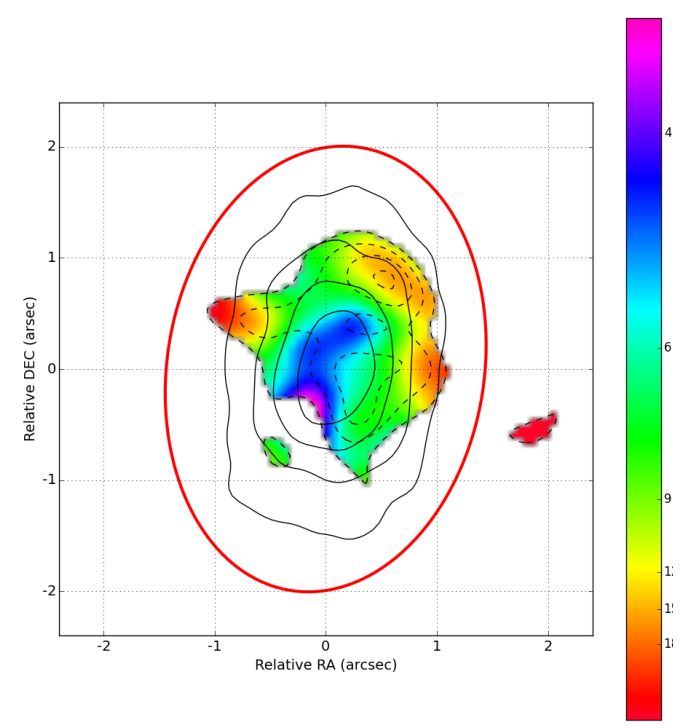

Fig. 3. Map of the optical depth of ${ }^{12} \mathrm{CO}$, the colour map is plotted for regions of the disc where we detect ${ }^{13} \mathrm{CO}>3 \sigma_{13}$. Overlaid in solid black contours of $(10,20,30,40) \times \sigma_{12}\left(\sigma_{12}=0.064 \mathrm{Jy} \mathrm{km} \mathrm{s}^{-1}\right.$ beam $\left.{ }^{-1}\right)$ is the ${ }^{12} \mathrm{CO}$ integrated intensity map convolved with the larger beam and dashed black contours show ${ }^{13} \mathrm{CO}$ as in Fig. 2.

for the disc temperature of $50 \mathrm{~K}$ (van der Tak et al. 2007) ${ }^{3}$. Using lower temperatures does not change this ratio by more than a factor of two and ensures we used the minimum amount of $\mathrm{CO}(2-1)$, therefore calculating the minimum optical depth. We convolved both the ${ }^{12} \mathrm{CO}$ and ${ }^{13} \mathrm{CO}$ images with the synthesized beam of the ${ }^{13} \mathrm{CO}$ image in order to ensure the images have the same effective smoothing. Under the assumption that both lines have the same excitation temperature, the ratio of ${ }^{12} \mathrm{CO}(2-1)$ and ${ }^{13} \mathrm{CO}(2-1)$ emission is then used to compute the optical depths of both lines, following the method used in, e.g. Schwarz et al. (2016), and assuming the ratio of optical depths to follow the ISM abundance of 77. The obtained map (Fig. 3) shows that $\tau_{{ }^{12} \mathrm{CO}}$ ranges from 3 to 45 (Fig. 3). $\tau^{13} \mathrm{CO}$ is therefore in the range from 0.04 to 0.58 .

Figure 3 confirms ${ }^{12} \mathrm{CO}(2-1)$, and therefore also (3-2), to be optically thick throughout the disc, while the ${ }^{13} \mathrm{CO}$ is not. In central regions $\tau_{{ }^{12} \mathrm{CO}} \sim 2$ where ${ }^{12} \mathrm{CO}$ is strongest, reaching as high as 25 in outer regions where ${ }^{13} \mathrm{CO}$ is detected strongly. Once ${ }^{12} \mathrm{CO}$ becomes optically thick, it ceases to trace mass in the midplane because emission is coming from upper layers of the disc at greater scale heights. This may also explain why the asymmetry of the disc is so prominent in the ${ }^{13} \mathrm{CO}$ map, but not in the ${ }^{12} \mathrm{CO}$; the optically thick ${ }^{12} \mathrm{CO}$ emission is dominated by the temperature of the emitting layer, which increases closer to the star, so a combination of temperature and emitting surface area are responsible for the brightness of emission, rather than the fractional changes in gas density. However the optically thin ${ }^{13} \mathrm{CO}$ traces density more faithfully and provides a better indicator of the spatial distribution of gas in the disc, highlighting any asymmetries which may be hidden by temperature and surface area effects in ${ }^{12} \mathrm{CO}$. The radial profiles shown in Fig. 1 illustrate this effect: the azimuthally averaged flux drops sharply after the peak at around $\sim 30 \mathrm{au}$ for ${ }^{12} \mathrm{CO}$, but the ${ }^{13} \mathrm{CO}$ emission is still $>75 \%$ of the peak value until after $100 \mathrm{au}$. This is clearer in the NW/SE slices in Fig. 1 taken in the direction of the ${ }^{13} \mathrm{CO}$ peak. In green, the ${ }^{12} \mathrm{CO}$ peaks much closer to the star where temperature effects are more prominent $(\sim 30 \mathrm{au}$ in the NW slice), whilst the ${ }^{13} \mathrm{CO}$ peaks much further out in the disc at a deprojected distance

Available at home.strw. leidenuniv.nl/moldata/radex.html of $\sim 120 \mathrm{au}$. The slices also illustrate the magnitude of the asymmetry in the midplane gas, with a very strong ${ }^{13} \mathrm{CO}$ peak towards the north-west that is considerably stronger than the average flux at that separation. This demonstrates the importance of using $\mathrm{CO}$ isotopologues, as done here, to investigate the disc midplane, and the gas density distribution within it.

Having shown that the ${ }^{13} \mathrm{CO}$ emission is optically thin, we derive a lower limit on the mass of the gas disc using

$M_{\text {gas }}=\frac{4 \pi}{h v_{21}} \frac{F_{21} m \mathrm{~d}^{2}}{A_{21} x_{2}}\left[\frac{\mathrm{H}_{2}}{{ }^{12} \mathrm{CO}}\right]\left[\frac{{ }^{12} \mathrm{CO}}{{ }^{13} \mathrm{CO}}\right]$

where $\mathrm{F}_{21}$ is the integrated line flux from the $J=2-1$ transition of ${ }^{13} \mathrm{CO}, \mathrm{m}$ is the mass of the $\mathrm{CO}$ molecule, $\mathrm{A}_{21}$ the Einstein coefficient for the transition, $v$ is the frequency at which the transition occurs and $x$ is the fractional population of the upper level. We take the midplane temperature to be $20 \mathrm{~K}$ and assume ISM abundances of ${ }^{12} \mathrm{CO} /{ }^{13} \mathrm{CO}=77$ and ${ }^{12} \mathrm{CO} / \mathrm{H}_{2}=10^{-4}$ (Wilson \& Rood 1994) to give a total gas disc minimum mass of $(6.0 \pm 0.9) \times 10^{-4} M_{\odot}$. Uncertainties are due to the ALMA $15 \%$ flux calibration uncertainty. Like the dust mass, this value depends on disc temperature, for example adopting $T=50 \mathrm{~K}$ would give $(9.0 \pm 1.0) \times 10^{-4} M_{\odot}$. Sequestration of CO into icy bodies or conversion into more complex molecules can reduce gas-phase $\mathrm{CO}$ in the disc, decreasing the gas mass calculated by this method. Our minimum mass is two orders of magnitude larger than that calculated using the same method but with ${ }^{12} \mathrm{CO}$ as the mass tracer, $\sim 4.5 \times 10^{-6} M_{\odot}$ (White et al. 2016). SMA observations $\mathrm{CO}(1-0)$ emission gives a gas disc mass $1.05 \times 10^{-4} M_{\odot}$, comparable to the mass we derive here, however MCMC modelling of the data gave a slightly lower value of $3.8 \times 10^{-5} M_{\odot}$ (Flaherty et al. 2016). Thi et al. (2014) also find a similar order of magnitude by fitting Herschel gas line observations in the far-IR to simultaneous radiative transfer and chemical modelling, giving a range of $2.5-5 \times 10^{-4} M_{\odot}$. Each of these methods assume ${ }^{12} \mathrm{CO} / \mathrm{H}=10^{-4}$.

${ }^{12} \mathrm{CO}$ therefore appears to be underestimating disc mass, agreeing with our confirmation of the tracer's optical thickness. A similar scenario was found in $30 \mathrm{Myr}$ old hybrid disc HD 21997; Kospal et al. (2013) calculate a CO gas mass in the disc from the optically thin $\mathrm{C}^{18} \mathrm{O}$ line that was two orders of magnitude larger than that previously calculated assuming optically thin ${ }^{12} \mathrm{CO}$ emission (Moór et al. 2011).

Our observations do not detect $\mathrm{C}^{18} \mathrm{O}$, but from the rms noise in the integrated image we can derive an upper limit on the $\mathrm{C}^{18} \mathrm{O}$ gas mass and a corresponding estimate of the disc gas mass. Rms in the $\mathrm{C}^{18} \mathrm{O}$ image with natural weighting is $25 \mathrm{mJy}$ beam $^{-1}$, yielding a total gas mass estimate of $1.4 \times 10^{-4} M_{\odot}$ assuming $T=20 \mathrm{~K}$, ISM abundances and isotopic ratios. This value is higher than that traced by the optically thick ${ }^{12} \mathrm{CO}$, but much lower than the estimate based on the ${ }^{13} \mathrm{CO}$. In fact, the flux ratio between ${ }^{13} \mathrm{CO}$ and the upper limit on $\mathrm{C}^{18} \mathrm{O}$ flux is 31 , significantly higher than the typical ISM abundance of eight (Wilson \& Rood 1994). This implies that $\mathrm{C}^{18} \mathrm{O}$ is under abundant by at least a factor of 3.8. An explanation for this may be the isotope-selective photo-dissociation, known to deplete the less abundant isotopologues such as $\mathrm{C}^{18} \mathrm{O}$ in discs (Visser et al. 2009; Miotello et al. 2016). The higher column density of the more abundant isotopologue, in this case ${ }^{13} \mathrm{CO}$, means that it can self-shield from photodissociation more effectively. It is important to note that isotope-selective photodissociation cannot be the cause of the differences between the gas mass estimates based on ${ }^{12} \mathrm{CO}$ and ${ }^{13} \mathrm{CO}$, as it would have the opposite effect: The mass based on ${ }^{12} \mathrm{CO}$ should be higher in such case. In case of optically thick ${ }^{12} \mathrm{CO}$ emission, as here, only the comparisons between the optically thin isotopologue lines allows us to assess the selective photodissociation effects, again stressing the importance of observing these species. 


\subsection{Nature of the disc}

In this section we compare our new results on disc structure and mass of gas and dust to some similar systems.

Known hybrid discs generally have disc gas masses ${ }^{4}$ derived from $\mathrm{CO}$ observations of order one Earth mass (e.g. 49 Ceti $6.6 \times 10^{-6} M_{\odot}$, HD $1318351.3 \times 10^{-5} M_{\odot}$, Hughes et al. 2008 ; Moór et al. 2015). HD 141569 has a particularly massive gas disc in comparison (see e.g. Fig. 6 of Péricaud et al. 2017). All previous estimates were based on optically thick tracers, which explains why in this work we derive $(6.0 \pm 0.9) \times 10^{-4} M_{\odot}$, in other words, two orders of magnitude greater mass. A more direct comparison can be made with hybrid disc HD 21997, where the disc mass derived from ALMA observations of optically thin $\mathrm{C}^{18} \mathrm{O}$ giving $0.8-1.8 \times 10^{-4} M_{\odot}$ (Kospal et al. 2013) a factor of a few less than HD 141569. Hybrid discs in general may be hosting more gas than is traced by $\mathrm{CO}$ and its isotopologues, not only due to optical depth but also if $\mathrm{CO}$ has been depleted with respect to $\mathrm{H}_{2}$.

The minimum dust mass $1.2 \pm 0.2 M_{\oplus}$ corresponding to the unresolved component is greater than the dust masses of HD 131835 (0.47 $M_{\oplus}$ Moór et al. 2015), HD $21997\left(0.09 M_{\oplus}\right.$ Moór et al. 2013) and 49 Ceti (0.2 $M_{\oplus}$ Holland et al. 2017).

The millimetre ring at 220 au has a minimum dust mass of $0.13 \pm 0.02 M_{\oplus}$. This mass and the scattered light ring seen at a slightly larger distance are both comparable to typical debris discs. Debris systems show evidence of rings containing millimetre sized grains at large radii outside an inner debris component like HD 181327 (Marino et al. 2016) and HD 107146 (Ricci et al. 2015; Marino et al. 2018), perhaps the nesting of structure within a larger ring is a more common component than we currently anticipate.

\section{Conclusions}

We detect an asymmetric distribution of midplane gas in the young hybrid disc HD 141569, traced by optically thin ${ }^{13} \mathrm{CO}$ emission. We also detect a faint mm ring of emission at $220 \pm 10$ au, providing a midplane counterpart to the ring detected in scattered light, where these small dust grains originate. We derive the minimum dust mass in the ring of $0.13 \pm 0.02 M_{\oplus}$, around $10 \%$ of the total flux from the system. The bulk of the millimetre emission, known to be variable, coincides with the location of the star and is unresolved, placing an upper limit on its radial extension of $72 \mathrm{au}$. This emission traces a minimum of $1.2 \pm 0.2 M_{\oplus}$ of dust, but may have a free-free contribution of $\leq 3 \%$. From our observations we are able to place an upper limit on the outermost ring of $0.11 \pm 0.02 M_{\oplus}$.

The gas disc is interior to this ring at $220 \mathrm{au}$. From the integrated ${ }^{13} \mathrm{CO}$ flux we derive a gas mass for the disc of $(6.0 \pm 0.9) \times 10^{-4} M_{\odot}$; a mass of approximately two orders of magnitude greater than that derived using ${ }^{12} \mathrm{CO}$. We show that the reason for this is the high optical depth of ${ }^{12} \mathrm{CO}$, rendering any gas mass value derived from its emission a large underestimate. Non-detection of $\mathrm{C}^{18} \mathrm{O}$ shows that this isotopologue is about four times less abundant than we would expect, based on the standard isotopic ratio between ${ }^{16} \mathrm{O}$ and ${ }^{18} \mathrm{O}$, and we propose the isotope-selective photodissociation as the culprit.

At the time of completion of the work on our data, the detection of the continuum ring was presented in a publication by others (White \& Boley 2018). We nonetheless present the analysis of our entire dataset, including both gas lines and the dust.

\footnotetext{
4 We assumed here ${ }^{12} \mathrm{CO} / \mathrm{H}_{2}=10^{-4}$ to convert $\mathrm{CO}$ masses to total disc masses in order for ease of comparison. For individual discs this ratio may be less appropriate than others.
}

Acknowledgements. We thank the anonymous referee for a valuable review. We would also like to thank Illaria Pascucci, Jane Greaves and Anita Richards for useful discussions. The work of J. M. is supported through the University Research Scholarship of the University of Leeds. The work of O. P. is funded by the Royal Society through the Dorothy Hodgkin Fellowship. G. M. K. is supported by the Royal Society as a University Research Fellow.

\section{References}

Ardila, D. R., Lubow, S. H., Golimowski, D. A., et al. 2005, ApJ, 627, 986 Augereau, J. C., \& Papaloizou, J. C. B. 2004, A\&A, 414, 1153 Augereau, J. C., Lagrange, A. M., Mouillet, D., \& Ménard, F. 1999, A\&A, 350, 51

Biller, B. A., Liu, M. C., Rice, K., et al. 2015, MNRAS, 450, 4446 Birnstiel, T., Dullemond, C. P., \& Pinilla, P. 2013, A\&A, 550, L8 Boehler, Y., Ricci, L., Weaver, E., et al. 2017, ApJ, 853, 162

Dent, W. R. F., Greaves, J. S., \& Coulson, I. M. 2005, MNRAS, 359, 663 Dent, W. R. F., Wyatt, M. C., Roberge, A., et al. 2014, Science, 343, 1490 Dominik, C., \& Dullemond, C. P. 2002, in The Origin of Stars and Planets: The VLT View (Heidelberg: Springer), 439

Draine, B. T. 2006, ApJ, 636, 1114

Flaherty, K. M., Hughes, A. M., Andrews, S. M., et al. 2016, ApJ, 818, 97

Goto, M., Usuda, T., Dullemond, C. P., et al. 2006, ApJ, 652, 758

Holland, W. S., Matthews, B. C., Kennedy, G. M., et al. 2017, MNRAS, 470, 3606

Hughes, A. M., Wilner, D. J., Kamp, I., \& Hogerheijde, M. R. 2008, ApJ, 681, 626

Jackson, A. P., Wyatt, M. C., Bonsor, A., \& Veras, D. 2014, MNRAS, 440, 3757 Kennedy, G. M., \& Wyatt, M. C. 2014, MNRAS, 444, 3164

Konishi, M., Grady, C. A., Schneider, G., et al. 2016, ApJ, 818, L23

Kospal, A., Moor, A., Juhasz, A., et al. 2013, ApJ, 776, 77

Lindegren, L., Lammers, U., Bastian, U., et al. 2016, A\&A, 595, A4

MacGregor, M. A., Wilner, D. J., Chandler, C., et al. 2016, ApJ, 823, 79

Marino, S., Matrà, L., Stark, C., et al. 2016, MNRAS, 460, 2933

Marino, S., Carpenter, J., Wyatt, M. C., et al. 2018, MNRAS, submitted

Marsh, K. A., Silverstone, M. D., Becklin, E. E., et al. 2002, ApJ, 573, 425

Matrà, L., Dent, W. R. F., Wyatt, M. C., et al. 2017, MNRAS, 464, 1415

Miotello, A., van Dishoeck, E. F., Kama, M., \& Bruderer, S. 2016, A\&A, 594, A85

Moór, A., Abrahám, P., Juhász, A., et al. 2011, ApJ, 740, L7

Moór, A., Juhász, A., Kóspál, A., et al. 2013, ApJ, 777, L25

Moór, A., Henning, T., Juhász, A., et al. 2015, ApJ, 814, 42

Panic, O., Holland, W. S., Wyatt, M. C., et al. 2013, MNRAS, 435, 1037

Péricaud, J., Di Folco, E., Dutrey, A., Guilloteau, S., \& Piétu, V. 2017, A\&A, 600, A62

Perrot, C., Boccaletti, A., Pantin, E., et al. 2016, A\&A, 590, L7

Pinilla, P., Birnstiel, T., Ricci, L., et al. 2012, A\&A, 538, A114

Pinilla, P., Benisty, M., Birnstiel, T., et al. 2014, A\&A, 564, A51

Rau, U., \& Cornwell, T. J. 2011, A\&A, 532, A71

Reche, R., Beust, H., \& Augereau, J.-C. 2009, A\&A, 493, 661

Ricci, L., Carpenter, J. M., Fu, B., et al. 2015, ApJ, 798, 124

Roberge, A., Kamp, I., Montesinos, B., et al. 2013, ApJ, 771, 69

Sandell, G., Weintraub, D. A., \& Hamidouche, M. 2011, ApJ, 727, 26

Schwarz, K. R., Bergin, E. A., Cleeves, L. I., et al. 2016, ApJ, 823, 91

Sylvester, R. J., Skinner, C. J., Barlow, M. J., \& Mannings, V. 1996, MNRAS, 279,915

Takeuchi, T., \& Artymowicz, P. 2001, ApJ, 557, 990

Takeuchi, T., \& Lin, D. N. C. 2005, ApJ, 623, 482

Thi, W. F., Pinte, C., Pantin, E., et al. 2014, A\&A, 561, A50

van der Marel, N., van Dishoeck, E. F., Bruderer, S., et al. 2013, Science, 340, 1199

van der Tak, F. F. S., Black, J. H., Schöier, F. L., Jansen, D. J., \& van Dishoeck, E. F. 2007, A\&A, 468, 627

Visser, R., van Dishoeck, E. F., \& Black, J. H. 2009, A\&A, 503, 323

Weinberger, A. J., Becklin, E. E., Schneider, G., et al. 1999, ApJ, 525, 53

Weinberger, A. J., Rich, R. M., Becklin, E. E., Zuckerman, B., \& Matthews, K. 2000, ApJ, 544, 937

White, J. A., \& Boley, A. C. 2018, ApJ, 859, 103

White, J. A., Boley, A. C., Hughes, A. M., et al. 2016, ApJ, 829, 6

White, J. A., Boley, A. C., MacGregor, M. A., Hughes, A. M., \& Wilner, D. J. 2018, MNRAS, 474, 4500

Wilson, T. L., \& Rood, R. T. 1994, ARA\&A, 32, 191

Wright, C. M., Maddison, S. T., Wilner, D. J., et al. 2015, MNRAS, 453, 414

Wyatt, M. C. 2003, ApJ, 598, 1321

Wyatt, M. C. 2005, A\&A, 440, 937

Wyatt, M. C., Panić, O., Kennedy, G. M., \& Matrà, L. 2015, Ap\&SS, 357, 103 